\title{
A note on Hausdorff groups
}

\section{Temple H. Fay}

It is well known that in the category of Hausdorff groups continuous homomorphisms having dense image are epic. That these homomorphisms are precisely the epics is a conjecture. If one could prove that proper closed subgroups (endowed with the subspace topology) cannot have epic inclusions, then the conjecture would be shown to be true. Closed subgroups fall into two classifications:

(a) open (and hence closed) subgroups and

(b) nowhere dense closed subgroups.

In this note it is shown that proper open subgroups cannot have epic inclusions. To do so an algebraic construction of Peter $J$. Hilton is topologized. The nowhere dense case remains open.

It is well known that in the category of Hausdorff groups continuous homomorphisms having dense image are epic. It is a long standing conjecture that these homomorphisms are precisely the epics. If $f: \vec{G} \rightarrow G$ is epic, then the inclusion map from the closure of $f(\bar{G})$ in $G$ is epic also. There is no loss of generality, therefore, to consider closed subgroups (endowed with the subspace topology) and the associated inclusion maps. Closed subgroups fall into two classifications:

(a) open (and hence closed) subgroups; and

(b) nowhere dense closed subgroups.

It will be shown that proper open subgroups cannot have epic inclusions. To do so an algebraic construction of Hilton [3] is topologized. The

Received 29 April 1975. The author wishes to express his thanks to Professor B.V.S. Thomas for pointing out this open problem. 
nowhere dense case remains open.

THEOREM. If $B$ is a proper open subgroup of a Hausdorff group $G$, then the inclusion $i_{H}: H \rightarrow G$ is not epic.

Proof. Endow the set of right cosets $G / H$ with the quotient topology induced by the canonical surjection $\eta$. Note $G / H$ is not necessarily a group. Because $H$ is open, $G / H$ is discrete.

Consider $\left(Z_{2},+\right)$ with the discrete topology; $Z_{2}$ is then a Hausdorff group. Let $Y=Z_{2}^{G / H}=\left\{f: G / H \rightarrow Z_{2} \mid f\right.$ is a function $\}$. With the compact-open topology, $Y$ is homeomorphic to $\prod_{x \in G / H}\left(Z_{2}\right)_{x}$ and hence is a Hausdorff group.

Endow $G \times Y$. with the product topology and define $\psi: G \times Y+Y$ by $\psi(g, f)=h$ where $h(H y)=f(H y g)$ for each $H y \in G / H$. To verify $\psi$ is continuous, it suffices to show that $\pi_{x} \psi$ is continuous for each projection $\pi_{x}$. For $H y=x$, define $m_{y}: G \rightarrow G$ by $m_{y}(g)=y g$. Let $e:(G / H) \times Y+Z_{2}$ be the evaluation map; that is, $e(x, f)=f(x)$. Since $G / H$ is locally compact, $e$ is continuous. It follows that $e(n \times I)\left(m_{y} \times I\right)=\pi_{x} \psi$ and thus $\psi$ is continuous.

For $(f, g),\left(f^{\prime}, g^{\prime}\right) \in Y \times G$, define

$$
(f, g) *\left(f^{\prime}, g^{\prime}\right)=\left(f+\psi\left(g, f^{\prime}\right), g g^{\prime}\right) \text {. }
$$

Since $\psi$ is continuous, $(Y \times G, *)$ with the product topology, is a Hausdorff group. This semidirect product shall be denoted $Y \circ G$.

Let $\chi: G / H \rightarrow Z_{2}$ be defined by $\chi(H)=1$ and $\chi(H y)=0$ for all $H y \neq H$. Let $k: G+Y$ be the constant map $k(g)=\chi$. The map $\{1, k\}: G \rightarrow G \times Y$ is continuous. Define $h: Y \rightarrow Y$ by $h(f)=\chi-f ; h$ is continuous. Hence $\sigma=h \psi\{1, k\}: G \rightarrow Y$ is continuous.

Define $\alpha, \beta: G \rightarrow Y \times G$ by $\alpha(g)=(0, g)$ and $\beta(g)=(\chi-\psi(g, \chi), g)$. Then $\alpha$ is a continuous homomorphism and $\beta$ is a homomorphism which is also continuous since $B=\{\sigma, 1\}$. It follows that $\alpha$ and $B$ are distinct and agree precisely on $H$. Thus $i_{H}$ cannot be epic. 
Nummela and Hofmann have shown that epic implies dense for the category of Hausdorff groups with equal uniformities. Other results for various categories of groups may be found in [4], [5], and [6].

\section{References}

[1] James Dugundji, Topology (Allyn and Bacon, Boston, 1966).

[2] Edwin Hewitt and Kenneth A. Ross, Abstract harmonic analysis, Volume I (Die Grundlehren der mathematischen Wissenschaften, 115. Academic Press, New York; Springer-Verlag, Berlin, Göttingen, Heidelberg, 1963).

[3] Peter J. Hilton, Category theory (Notes from a National Science Foundation Short Course, Colgate University, Hamilton, New York, 1972).

[4] W.F. LaMartin, "On the foundations of k-group theory", preprint.

[5] W.F. LaMartin, "Epics in the category of $T_{2} k$-groups need not have dense range", preprint.

[6] Detlev Poguntke, "Epimorphisms of compact groups are onto", Proc. Amer. Math. Soc. 26 (1970), 503-504.

\footnotetext{
Department of Mathematics, Hendrix College, Conway, Arkansas, USA.
} 